\title{
Optimization of triple inverted pendulum control process based on motion vision
}

\author{
Xiaoping Huang ${ }^{1 *}$, Fangyi Wen ${ }^{2}$ and Zhongxin $\mathrm{Wei}^{2}$
}

\begin{abstract}
An inverted pendulum is a typical nonlinear and absolutely unstable system. In order to control the triple inverted pendulum effectively and steadily, an optimization method of inverted pendulum control process based on motion vision was proposed. The real-time motion pictures of the triple inverted pendulum in the swinging-up process were collected through the CCD camera, and the real-time motion pictures of the triple inverted pendulum were recognized, matched and optimized by using Harris algorithm. By using motion vision to control and optimize the triple inverted pendulum, the stabilization control of the triple inverted pendulum was realized.
\end{abstract}

Keywords: Motion vision, Triple inverted pendulum, Control process optimization

\section{Introduction}

The inverted pendulum is the model of the most balanced control and the lack of driving control system, which is widely used in teaching and theoretical research. Therefore, for many years, people have been working on the problem of inverted pendulum control in theory and function. The theory of control operation and the method of control will have a great promotion and positive significance for the industrial production. Automatic control is a highly theoretical subject and it is a very important engineering technology. The engineering technology theory mentioned in the literature [1] was "automatic control theory." In the scope of the improvement of the control theory, it is not feasible to use the theory in reality without proving the correctness of a theory. It is necessary to design the controller according to its theory in order to achieve the true value of the theory $[2,3]$. In literature [4], it was pointed out that the inverted pendulum, as a target of experiment, is a system with multiple variables, rapid transformation, nonlinearity, and poor stability. We can add a control algorithm to increase its stability to prove the processing ability of the control method for complex, unstable, and nonlinear systems. At the same

\footnotetext{
* Correspondence: nnxyhxp@126.com; 82201232@qq.com;

1355645755@qq.com

${ }^{1}$ College of Mechanical and Electrical Engineering, Nanning University, No.8 Lonting Road, Nannin 530200, China

Full list of author information is available at the end of the article
}

time, how this method embodies the difficult problems in a series of automatic control categories, such as robustness, stabilization, and tracking in the process of controlling the system, could also be reflected in the process of controlling the inverted pendulum movement. Therefore, it was concerned by the scientists. Using different control algorithms, different types of inverted pendulum control have become a worldwide research topic with high research value $[5,6]$.

The fuzzy control theory was used to control the inverted pendulum in the literature [7], when the fuzzy control method was used to analyze the inverted pendulum problems, the mathematical theory was not used to analyze the nonlinear factors in the inverted pendulum system and the influence of them on the controller, but was used to analyze all kinds of unstable conditions caused by the method in the control of the inverted pendulum, according to the controller's experience and the previous experience. Finally, the mode of the fuzzy control theory set was used to express the processing methods. However, the fuzzy control theory did not have a concise and practical way to deal with multivariable problems. Therefore, the analysis of the triple inverted pendulum system was not entirely applicable.

The neural network was applied to the inverted pendulum control system and developed rapidly in the early 1990s. It was mentioned in the literature 
[8] that the neural network could arbitrarily approach the complex nonlinear relationship as much as possible, could learn to train data samples and was suitable for the absolutely unstable inverted pendulum control system, and could use the characteristics that all the characteristic information with a certain quantity or a certain nature could be evenly stored in each layer of neurons in the network, so this method had strong robustness and capacity. The back-propagation neural network could also be combined with the $\mathrm{Q}$ learning algorithm, and was used as the non-discrete model to control learning of inverted pendulum. However, due to the sensitivity of the neural network system and slow convergence rate, the computation of objective function was very complex and time consuming.

In view of the incomplete research of the inverted pendulum control system by the above methods, the triple inverted pendulum control system based on visual difference feedback was proposed. The vision sensor camera was used to collect the image information during the inverted pendulum motion, and the image corner matching was applied to monitor the inverted pendulum in real time. Then, the mathematical model of the triple inverted pendulum control system was constructed, and the balance control of the inverted pendulum system was carried out through the linear quadratic optimal control theory $[9,10]$.

\section{Methods-optimization of triple inverted pendulum system control process}

\subsection{Inverted pendulum control system of motion vision}

After collecting the motion image information of triple inverted pendulum by visual sensor, we set up an application network remote control platform. The triple inverted pendulum was mainly composed of car, pendulum 1 , pendulum 2 , and pendulum 3 , and there were free links between them. The car could move horizontally on the horizontal guide rail, and the pendulum bar could move freely in the vertical plane. The motion analysis of the system diagram was shown in Fig. 1.

Firstly, we used a camera to collect image information of inverted pendulum and make the image preprocessing. Then, we selected the annotation template and placed it in the same plane, collected the template images from different angles, detected the feature points (corner points) in the image, obtained the internal and external parameters of the camera, and optimized the refinement.

Secondly, we extracted the image feature point information. The corner detection method was based on Harris corner monitoring and matching method.

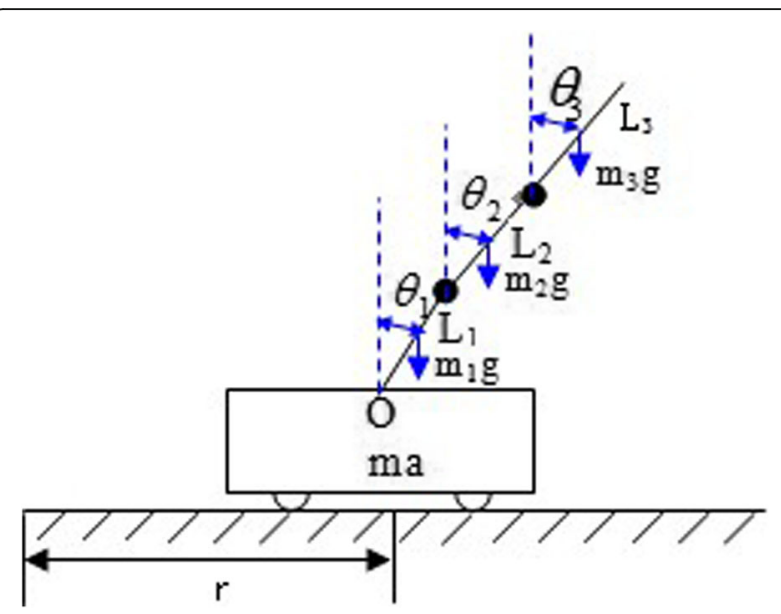

Fig. 1 Schematic diagram of the three level inverted pendulum

For the pixels $(x, y)$ on the image, the image window $W(W(x, y)$ was the image window function) should be moved on the quantification $(u, v)$. If we wanted to define the energy function $E$, the formula (1) was shown as follows.

$$
E(u, v)=\sum_{x, y} W(x, y)[I(x+u, y+v)-I(x, y)]^{2}
$$

The Harris corner was based on the grayscale images, so $I(x, y)$ was represented the gray value of images. Taylor expansion method was applied to deal with small quantities, so when $(u, v)$ was small, $E$ could be represented by the following formula (2).

$$
E(u, v) \approx[u, v] M\left[\begin{array}{l}
u \\
v
\end{array}\right]
$$

Among formula (3),

$$
\begin{aligned}
& M=\sum_{x, y} W(x, y) \otimes\left[\begin{array}{cc}
I_{x}^{2} & I_{x} I_{y} \\
I_{x} I_{y} & I_{y}^{2}
\end{array}\right]= \\
& {\left[\begin{array}{cc}
\sum_{x, y} G \otimes I_{x}^{2} & \sum_{x, y} G \otimes I_{x} I_{y} \\
\sum_{x, y} G \otimes I_{x} I_{y} & \sum_{x, y} G \otimes I_{y}^{2}
\end{array}\right]}
\end{aligned}
$$

$I_{x}=\frac{\partial I}{\partial x}$ and $I_{y}=\frac{\partial I}{\partial y} \quad$ respectively represented the first-order differential step degree of the image in the direction of $x$ and $y$. The image had noise when it was obtained and conveyed, so there were many methods to increase the level of noise resistance. Gauss smoothing filter for anti-noise processing was used in this paper, and $W$ was the Gauss template function. The expression was shown in formula (4). 


$$
W(x, y)=G=\exp \left[-\frac{1}{2 \sigma^{2}}\left(x^{2}+y^{2}\right)\right]
$$

$E$ was close to local autocorrelation function. Assuming that $\lambda 1$ and $\lambda 2$ were the eigenvalues of matrix $\mathrm{M}$, we could use $\lambda 1$ and $\lambda 2$ to determine the corner. $R$ was defined as the interest value of the corresponding pixel points; the formula was shown as follows in formula (5).

$$
R=\operatorname{det} \mathrm{M}-k(\text { trace } \mathrm{M})^{2}
$$

Among them, $k$ was the constant, and according to the experience, the value was $0.04-0.06$, trace $M=\lambda 1$ $+\lambda 2$ represented the direct trace of the matrix, and det $M=\lambda 1 \lambda 2$ was the determinant of the matrix. Then, the basis for obtaining Harris corner was that the $R$ value of a pixel in a frame was the best in other areas, which was greater than the set threshold $R_{0}$.

There was a phenomenon of rotation and deviation in the two images. Under the condition of obtaining the corner with the same threshold $R_{0}$, if the corner set of the overlapping parts of the image was set to $A$ and $A^{\prime}$, the necessary condition for the image matching was given first:

1. The number of corners should be the same, $A$ $=\left\{a_{1}, a_{2}, \ldots, a_{n}\right\}$ and $A^{\prime}=\left\{a_{1}{ }^{\prime}, a_{2}{ }^{\prime}, \ldots, a_{n}{ }^{\prime}\right\}$ had the same number of corners;

2. $a_{1}$ and $a_{1}{ }^{\prime}$ were the corresponding corners, then they had the same corner value $R\left(a_{1}\right)=r\left(a_{1}{ }^{\prime}\right)$;

3. If $a_{1}$ and $a_{1}{ }^{\prime}$ were the corresponding corner points, their number of adjacent corner points should be equal, that was $N_{r}\left(a_{1}\right)=N_{r}\left(a_{1}{ }^{\prime}\right), r$ represented the neighborhood radius;

4. If $\left\{a_{1}, a_{\mathrm{i}}\right\} \varepsilon A,\left\{a_{1}{ }^{\prime}, a_{\mathrm{i}}{ }^{\prime}\right\} \varepsilon A$ were the corresponding corner point, then their distance was equal $\mathrm{d}\left(a_{1}, a_{i}\right)$ $=d\left(a_{1}{ }^{\prime}, a_{i}{ }^{\prime}\right)$;

5. If $a_{i}$ and $a_{j}$ were corresponding corner points, $a_{i}{ }^{\prime}$ and $a_{j}{ }^{\prime}$ were other corresponding corner points, $\left\{a_{1}, a_{\mathrm{i}}\right\} \varepsilon A,\left\{a_{1}{ }^{\prime}, a_{i}{ }^{\prime}\right\} \varepsilon A$. According to $a_{i}, a_{j}, a_{i}{ }^{\prime}$, and $a_{j}{ }^{\prime}$, the matching parameter was calculated by the following formula (6).

$$
P_{i j}=\left(\Delta x_{i j}, \Delta y_{i j}, \Delta \theta_{i j}\right)
$$

$\Delta x_{i j}$ and $\Delta y_{i j}$ were the translational quantities of the image waiting for matching for the matching image on $x$ and $y$ axis, $\Delta \Theta_{i j}$ was the angle of rotation. If the matching parameter $P_{i j}$ of any matching corner point in the $A$ and $A^{\prime}$ set was the constant vector, then the two images matched completely.

\subsection{Motion control optimization of triple inverted pendulum system}

Firstly, we constructed the mathematical model of triple inverted pendulum system according to Lagrange equation and set $r$ as the motion displacement of horizontal guide rail, and $\theta_{1}, \theta_{2}$, and $\theta_{3}$ were respectively the angles of the lower, middle, and upper pendulums relative to the vertical direction. The Lagrange equation for the triple inverted pendulum system with conservative force and dissipative force were shown in the following formula (7).

$$
\frac{d}{d t}\left(\frac{\partial T}{\partial \dot{q}_{i}}\right)-\frac{\partial T}{\partial \dot{q}_{i}}+\frac{\partial V}{\partial q_{i}}+\frac{\partial D}{\partial \dot{q}_{i}}=F_{q_{i}}
$$

In the formula, $q_{i}$ represented the generalized coordinate, that were $r, \Theta_{1}, \Theta_{2}, \Theta_{3} ; F_{\mathrm{qi}}$ was the non-potential generalized force, when $q_{i}=r, F_{q i}=G_{0} U, U$ was the controlled quantity, $G_{0}$ and was the gain constant. When $q_{i}$ $=\Theta_{1}, \Theta_{2}$, and $\Theta_{3}, F_{q i}=0, T, V$, and $D$ were respectively the function, potential energy, and dissipative energy of the system, then $T=\sum_{i=0}^{n} T_{i}, \quad V=\sum_{i=0}^{n} V_{i}$, and $D=\sum_{i=0}^{n} D_{i}$. Among them, $n$ was the stage number of the inverted pendulum $(n=3) ; T_{i}, V_{i}$, and $V_{i}$ were kinetic energy, potential energy, and dissipative energy of cars and all levels of pendulum. The mathematical model could be obtained by putting $T_{i}, V_{i}$ and $D_{i}(i=0,1,2,3)$ into the formula (8).

$$
\begin{aligned}
M\left(\theta_{1}, \theta_{2}, \theta_{3}\right)\left[\begin{array}{c}
r^{n} \\
\theta_{1}{ }^{n} \\
\theta_{2}{ }^{n} \\
\theta_{3}{ }^{n}
\end{array}\right]+ & N\left(\theta_{1}, \theta_{2}, \theta_{3}, \dot{\theta}_{1}, \dot{\theta}_{2}, \dot{\theta}_{3}\right) \\
& \times\left[\begin{array}{c}
r^{\prime} \\
\theta_{1}{ }^{\prime} \\
\theta_{2}{ }^{\prime} \\
\theta_{3}{ }^{\prime}
\end{array}\right]=G\left(u, \theta_{1}, \theta_{2}, \theta_{3}\right)
\end{aligned}
$$

Formula (8) was a nonlinear vector differential equation. When the system worked, it moved on the edge of the equilibrium position. In the formula, when $u=0$, it was linearized near the equilibrium position $r=\theta_{1}=\theta_{2}=\theta_{3}$ $=\dot{r}=\dot{\theta}_{1}=\dot{\theta}_{2}=\dot{\theta}_{3}=0$ instead of the nonlinear vector differential equation.

Secondly, a linear quadratic optimal control was introduced to improve the triple inverted pendulum system.

1. The pendulum bars were linked by hinges without any constraints, so it was difficult to control the attitude in the initial swing. Therefore, in order to control the state balance 


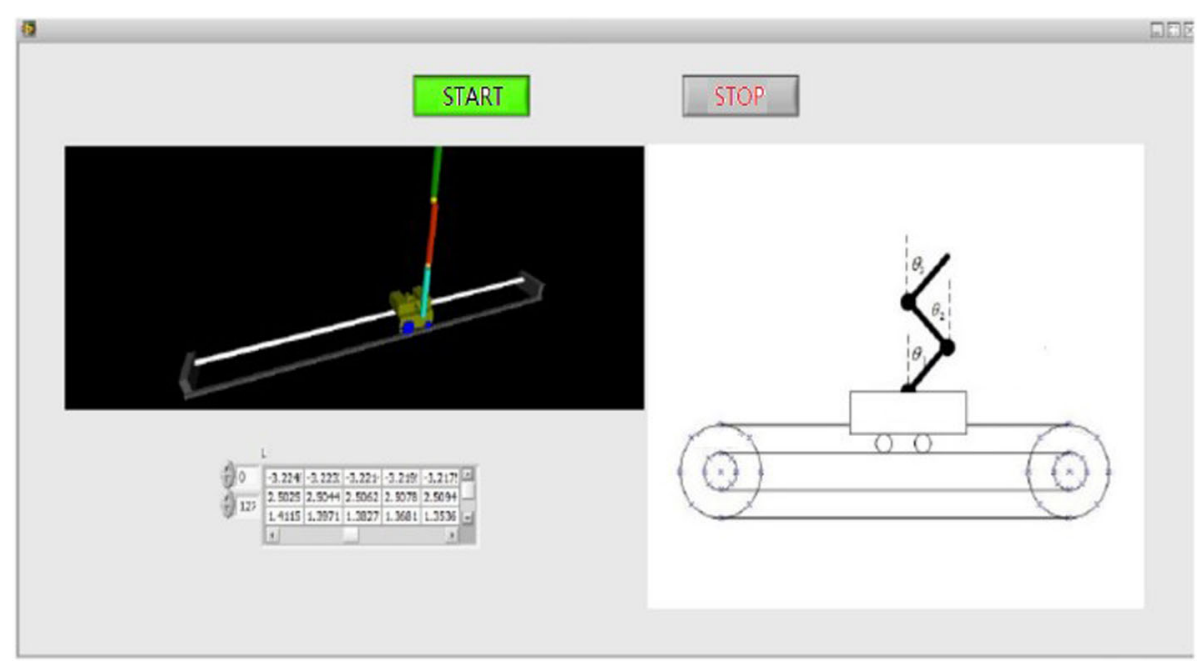

Fig. 2 3D animation simulation results of the control system

of the triple inverted pendulum in the initial swing process, we used the finite-time time invariant LQ (liner quadratic) problem to achieve the optimal control.

$$
\left\{\begin{aligned}
-\dot{P}(t)= & P(t) A+A^{T} P(t)+Q(t) \\
& -P(t) B R^{-1}(t) B^{T} P(t) \\
P\left(t_{f}\right)= & S, t \in\left[t_{0}, t_{f}\right]
\end{aligned}\right.
$$

In the formula (9), A and B were the coefficient matrices of the corresponding dimensions, $S$ was the weighting matrix, and $t$ was the adjusting time. The solution matrix $P(t)$ was $n \times n$ positive semi definite symmetric matrix, then the necessary and sufficient conditions of the optimal control $\dot{u}(\bullet)$ were shown in formula (10).

$$
\dot{u}(t)=-\dot{K}(t) \dot{x}(t), \dot{K}(t)=R^{-1} B P(t)
$$

The optimal trajectory $\dot{x}(\bullet)$ was shown in formula(11).

$$
\dot{x}(t)=A \dot{x}(t)+B \dot{u}(t), \dot{x}\left(t_{0}\right)=x_{0}
$$

The optimum performance value $\dot{J}=J(\dot{u}(\bullet))$ was shown in formula (12).

$$
\dot{J}=\frac{1}{2} x_{0}^{T} P\left(t_{0}\right) x_{0}, \forall x_{0} \neq 0
$$

The optimal control system was adjusted by finite-time time invariant LQ, and its control state was observed. The feedback was that the inverted pendulum was stabilized and the jitter time was short in the process from swinging up to steady state.
2. The other key link of the triple pendulum automatic swing was the stable state entering the inverted position after swinging up. This area was a balance control problem. If it could not be effectively controlled, it would lead to the failure of the whole control system. Therefore, the control tending to steady state was based on the infinite-time LQ to adjust the optimal control. The sufficient and necessary condition for the given infinite-time time invariant LQ to regulate optimal control was shown as follows in formula (13).

$$
\dot{u}(t)=-\dot{K}(t) \dot{x}(t), \dot{K}=R^{-1} B^{T} P
$$

The equation of state for the optimal trajectory $\dot{x}(t)$ was shown in formula (14).

$$
\dot{x}(t)=A \dot{x}(t)+B u, \dot{x}\left({ }_{0}\right)=x_{0}
$$

The optimal performance value $\dot{J}=J(\dot{u}(\bullet))$ was shown in formula (15).

$$
\dot{J}=x_{0}^{T} P x_{0}, \forall x_{0} \neq 0
$$

3. Two-point boundary value problem: The triple pendulum control system was in the limited time $t \in[0, \mathrm{~T}]$, and the following boundary value conditions was satisfied to the process from static sag to vertical inverted position. 


$$
\begin{aligned}
& \left.\mathrm{y}(\mathrm{t})\right|_{\mathrm{t}=0, \mathrm{~T}}=0,\left.\dot{\mathrm{y}}(t)\right|_{\mathrm{t}=0, \mathrm{~T}}=0 \\
& \theta(0)\left|=\left[\begin{array}{lll}
\pi & \pi & \pi
\end{array}\right]^{\mathrm{T}}, \dot{\theta}(t)\right|_{\mathrm{t}=0, \mathrm{~T}}=0
\end{aligned}
$$

Among them, $t=0$ was the initial value condition, and $t=\mathrm{T}$ was the final value condition. In addition, according to the performance of the hardware, and the high sensitivity and strong instability of the triple pendulum control system, the action of the car must also satisfy the following constraints during the hoisting process.

$$
|\mathrm{y}| \leq 1.5 \mathrm{~m},|\dot{y}| \leq 5 \mathrm{~m} / \mathrm{s},|\ddot{y}| \leq 20 \mathrm{~m} / \mathrm{s}^{2}
$$

$\bar{\theta}^{*}=\left[\begin{array}{ll}\theta^{*} & \dot{\theta}^{*}\end{array}\right]^{\mathrm{T}}=\left[\begin{array}{llllll}\theta_{1}^{*} & \theta_{2}^{*} & \theta_{3}^{*} & \dot{\theta}_{1}^{*} & \dot{\theta}_{2}^{*} & \dot{\theta}_{3}^{*}\end{array}\right]$ was appointed, the first-order ordinary differential equations could be obtained was shown in formula (19).

$$
\dot{\bar{\theta}}^{*}=\bar{\beta}\left(\theta^{*}, \dot{\theta}^{*}, \ddot{y}^{*}\right)=\left[\begin{array}{llllll}
\theta_{1}^{*} & \theta_{2}^{*} & \theta_{3}^{*} & \dot{\theta}_{1}^{*} & \dot{\theta}_{2}^{*} & \dot{\theta}_{3}^{*}
\end{array}\right]
$$

Where the vector function $\bar{\beta}=\left[\begin{array}{llllll}\bar{\beta}_{1} & \bar{\beta}_{2} & \bar{\beta}_{3} & \bar{\beta}_{4} & \bar{\beta}_{5} & \bar{\beta}_{6}\end{array}\right]^{\mathrm{T}}$, the edge value condition were shown in formula (19) and formula (20).

$$
\begin{aligned}
& \theta^{*}(0)=\left[\begin{array}{lll}
\pi & \pi & \pi
\end{array}\right]^{\mathrm{T}}, \dot{\theta}^{*}(0)=0 \\
& \theta^{*}(\mathrm{~T})=0, \dot{\theta}^{*}(T)=0
\end{aligned}
$$

Obviously, ordinary differential Eq. (19) and boundary value condition (20), (21) were formed a nonlinear two-point boundary value problem.

4. Reference ground track: The design method of the nominal trajectory $y^{*}=\gamma(t, p)$ was not unique, polynomial functions or cosine series could be selected. In general, the input $u^{*}(t)$ was required to be continuous, so $\gamma(t, p)$ must meet the demands of quadratic differential if $\alpha=2$.

We should combine the finite-time and infinite-time invariant LQ regulation to improve the control, so that the system could reduce the attitude adjustment time in the automatic swing-up transition process and could quickly enter the stable stage of the automatic swinging up. After entering the stable stage, the inverted control of the triple pendulum was also the key stage. Through the adjustment of the infinite-time time invariant LQ, the system would maintain a steady and gradual process in a state tending to equilibrium.

\section{Analysis of the result of optimization process}

The inverted pendulum system was a very unstable system. In order to stabilize the state of the automatic swing-up transition stage and steady swing phase during
Table 1 The automatic swing-up adjustment control schedule

\begin{tabular}{lll}
\hline Methods & $\begin{array}{l}\text { Moving distance of } \\
\text { pendulum }(\mathrm{cm})\end{array}$ & $\begin{array}{l}\text { Adjustment } \\
\text { time }(\mathrm{t} / \mathrm{s})\end{array}$ \\
\hline Method is this paper & 15 & 7 \\
Literature [7] method & 4 & 25 \\
\hline
\end{tabular}

the control process, the finite-time and infinite-time invariant LQ were used to adjust the optimal control to improve the triple inverted pendulum system in this paper. In order to prove the effectiveness of the improved method in the aspect of stability control, we carried out experiments in MATLAB simulation environment, and waw collected datum and analyzed them. In the process of control, the moving effect of the pendulum and car in the triple inverted pendulum was presented visually and vividly in the form of animation in Fig. 2. And we could observe that many directions control the motion of the rocker and the car in the process of the controller to control the whole process of mobile, and it was a very vivid triple inverted pendulum visual simulation platform.

\section{Results and discussions}

\subsection{Experiment 1}

In automatic swing-up transition phase, in order to control the accurate swing of cars and pendulum bars, the finite-time time invariant LQ was used to adjust the optimal control, then we could achieve the steady state of the pendulum bars in the initial swing process, after the system was adjusted by the method in the text and literature [7] method, they gradually reached a stable state. The following table was the control time data of automatic swing-up adjustment when the pendulum bars by two methods starts to swing was shown in Table 1.

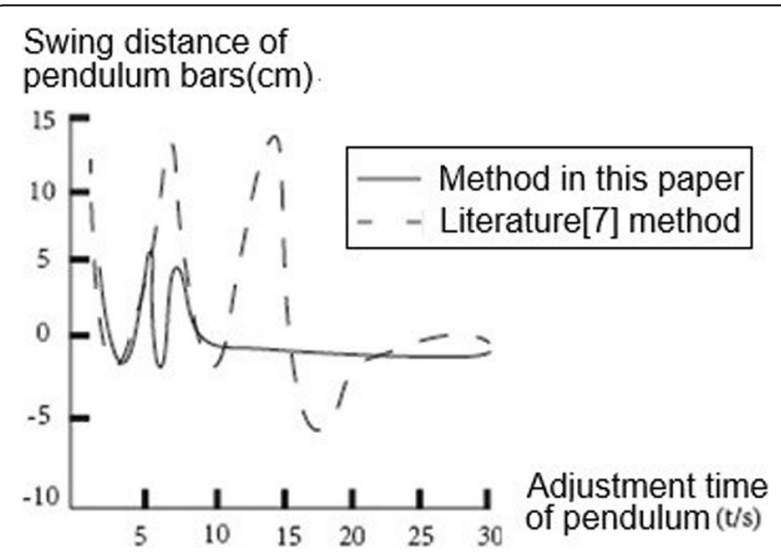

Fig. 3 Comparison of adjustment time curves of pendulum bars of two methods. 


\section{Pendulum bar radian ( $\mathrm{rad}$ )}

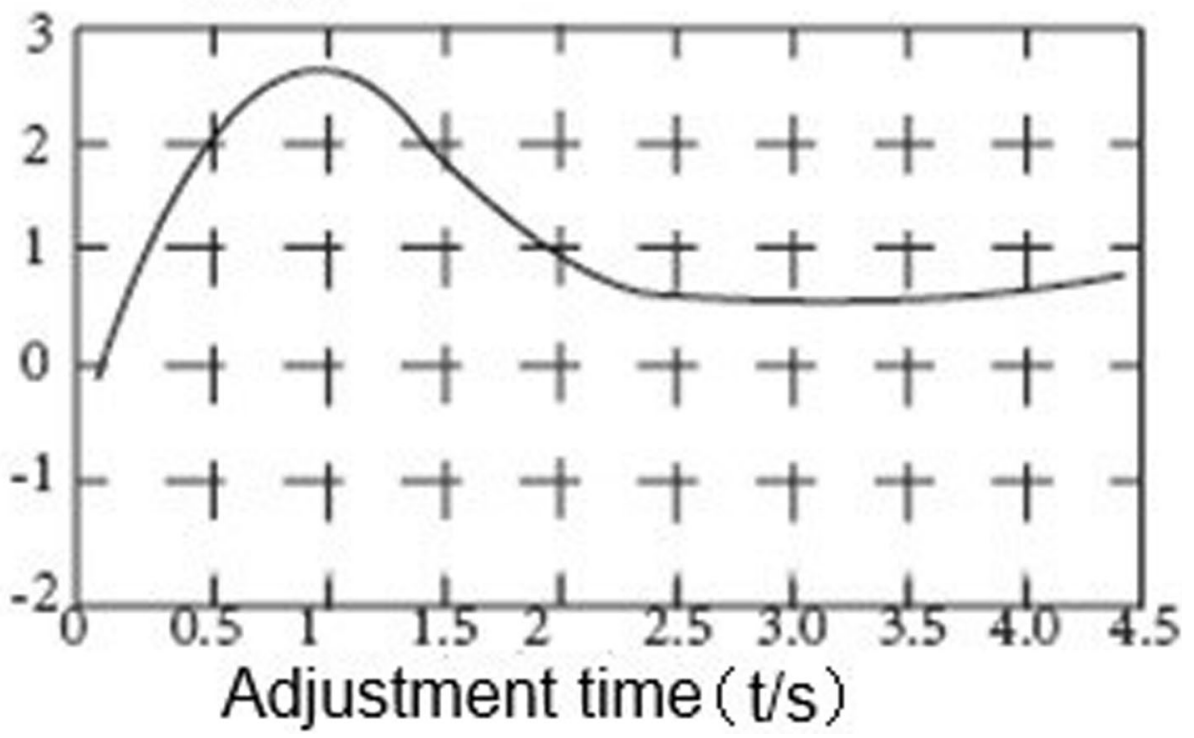

Fig. 4 The radian adjustment figure of the inverted pendulum by the method of literature

From data analysis of Table 1 , the two methods adjust the initial swing stage of the inverted pendulum simultaneously. It could be seen from the data that the adjustment time of the literature [7] method was too long, so the stability of the control system was not strong. According to Table 1, we could get the adjustment time curve figure of the inverted pendulum of two methods, as shown in Fig. 3.

From the two curves of Fig. 3, it was observed that the time consuming of the inverted pendulum from the initial pendulum to the steady motion of the pendulum bar was about 7-8 s, while the time consuming of the literature [7] was about $25 \mathrm{~s}$. Therefore, the control performance of the initial pendulum stage of the method in this paper was better.

In the process of inverted pendulum adjustment, the swing angle radian would be generated. From Figs. 3 to 4, we would analyze the swing angle radian in the initial swing time of the inverted pendulum controlled by two methods.

From Figs. 4 and 5, we could see the difference of the swing radian in the swinging-up stage of the inverted pendulum. After using the method in this paper to control the inverted pendulum, the swing angle radian of initial swing was much less than that of literature [7] method, and it was indicated that the method in this paper was effective for the triple inverted pendulum in the initial swing stage.

\subsection{Experiment 2}

Stable swing stage: For the stable swing stage of the triple inverted pendulum from initial swing stage to the steady motion, the swing state was the control of the balance. Figure 6 was the comparison of the optimal control curve given by the system and the curves of the steady swing stage of two methods.

The motion velocity of inverted pendulum in steady swing stage could be analyzed from Fig. 6, the motion

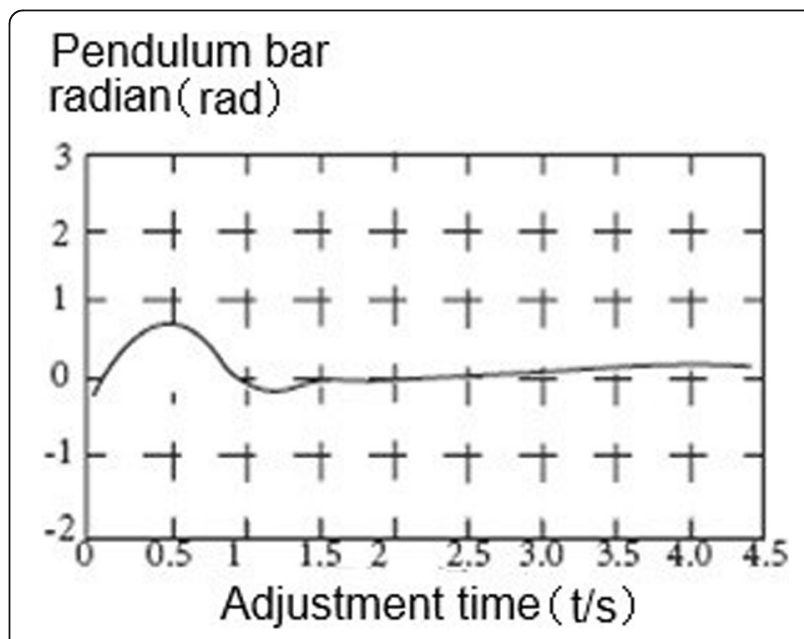

Fig. 5 The radian adjustment figure of the inverted pendulum by the method in this paper. 


\section{Optimal motion velocity $(\mathrm{m} / \mathrm{s})$}

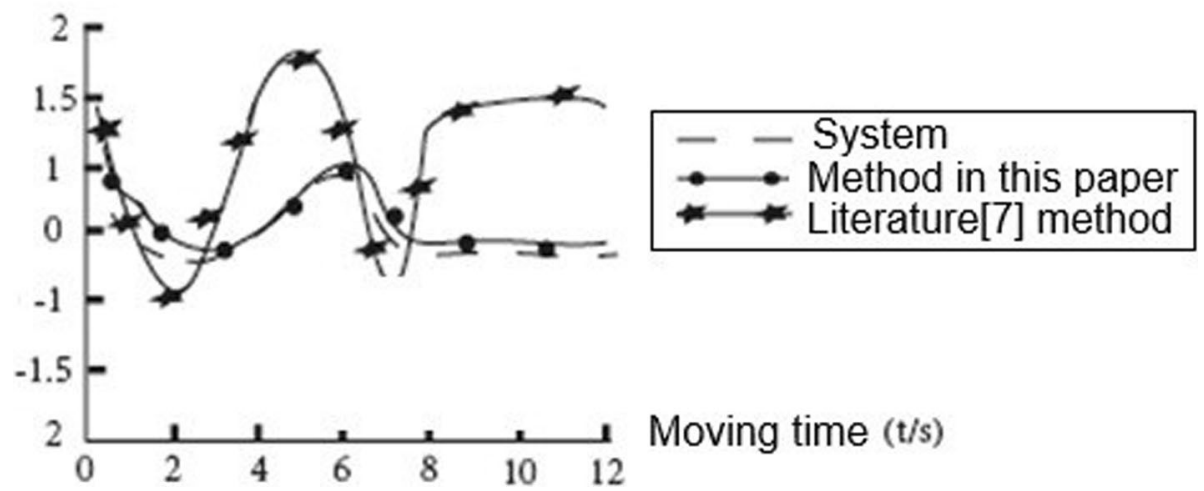

Fig. 6 Comparison chart of the movement velocity of the steady swing stage of the inverted pendulum

velocity of the inverted pendulum controlled by the method in this paper was close to the optimal speed of the system, and the goodness of fit was better. The motion speed of literature [7] algorithm was faster, and it was indicated that the inverted pendulum was always in a state of quivering.

\subsection{Experiment 3}

When we constructed the triple inverted pendulum control model, at first, we set an initial displacement angle for triple inverted pendulum, and the starting position of the three-stage inverted pendulum car was given by the simulation control system to the force $\mathrm{F}$ in a uniform direction at the bottom of the car. Within $20 \mathrm{~s}$, we recorded the motion state and the degree of pendulum and kept the car in a set of orbit range ( 0 to $10 \mathrm{~m}$ ), then kept the third movement that did not exceed the scope of the above a predetermined angle.

The initial object of the three-stage inverted pendulum system was set in the same range and randomly selected in the above range. Through constant updates, the control system could control the inverted pendulum, so the time of its movement was within the prescribed limits. The time period and sampling frequency were $0.5 \mathrm{~s}$ to ensure that the three-stage inverted pendulum could be maintained in a certain angle. In the case of white noise signal interference and shock signal interference, the stability of the system was maintained, and the robustness was strong. The range and control of the upper, middle and lower positions with white noise and impulse were shown in Fig. 7a-c.

With the control method of this paper, the amplitude of the three-stage inverted pendulum could be stabilized at a certain time. This method had the advantages of high modeling accuracy and good controllability, which could be used to solve the problems of complex systems which were difficult to solve by traditional methods.

\section{Conclusions}

In this paper, the triple inverted pendulum control system based on the motion vision was proposed, and motion attitude of the inverted pendulum was monitored by vision sensor. The finite-time and infinite-time time invariant LQ control method were introduced to control the balance of the triple inverted pendulum from the initial swing stage to the steady swing stage. The experiments showed that the inverted pendulum control system designed in this paper solved the problem of changeable and unstable
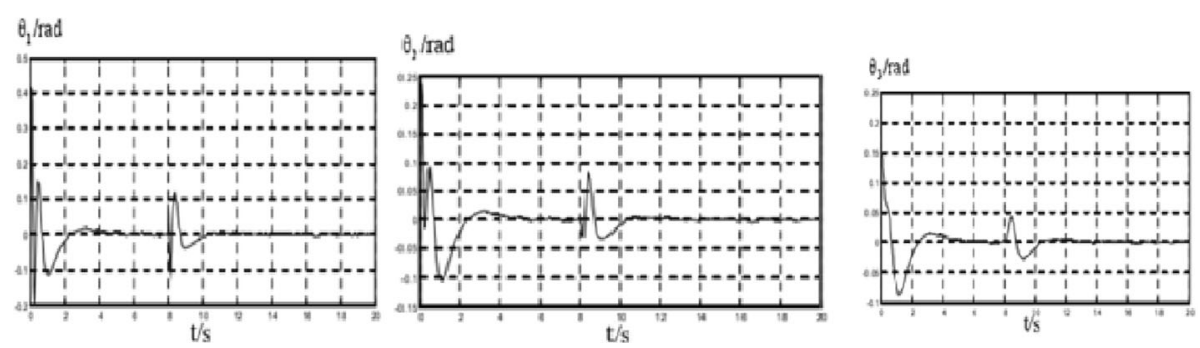

Fig. 7 The control curve of the triple inverted pendulum 
inverted pendulum. The parameters of the controller were further optimized on the basis of optimizing feedback control parameters; thus, we could improve the convergence speed of the system. The design of the controller accords with the goal and principle of triple inverted pendulum control system, which was an effective design scheme, and it could provide some reference value for other fields of the control theory.

\section{Abbreviations}

CCD: Charge coupled device; LQ: Liner quadratic

\section{Acknowledgements}

The authors thank the editor and anonymous reviewers for their helpful comments and valuable suggestions.

\section{Funding}

This work was supported in part by a grant from the Industry-Academy Cooperation Education Project of the Ministry of Education (project No.201702135097), the Science and Technology Planning Project of Guangxi Bureau of quality and technology supervision (contract No.GXKJJH2018009)

\section{Availability of data and materials}

The data and materials are available from the corresponding author on reasonable request.

\section{Authors' contributions}

All authors have taken part in the discussion of the work described in this paper. $\mathrm{XH}$ wrote the first version of the paper. FW took part in the experiments of the paper. ZW revised the paper in different versions. All authors read and approved the final manuscript.

\section{Authors' information}

Xiaoping Huang received the B.S. degree in automation from Hunan Industry University, Zhuzhou, China, in 1996, and the M.S. degree in Control Science and Engineering from Guilin University of Technology, Guilin, China, in 2007. From 1996 to 2010, he was a Senior engineer in Guilin Shuguang Rubber Industry Research and Design Institute. From 2011 to 2016, he was an associate professor of the college of Mechanical and Electrical Engineering at Nanning University. From 2017 to now, he was a professor of the college of Mechanical and Electrical Engineering at the same University. His research interests include Intelligent Control and Embedded System.

Fangyi Wen received her B.S. degree in Computer science and technology in Guilin University of Electronic Science and Technology in 2005, and she is currently a senior engineer at Guangxi Quality Technical Engineering School. Her current research interests focus on Computer Control Technology. Zhongxin Wei received his B. S. and M. S. degrees from Xi'an Jiaotong University in 1988 and 1991, he is currently engineer Senior engineer at Guangxi Quality Technical Engineering School. His current research interests include automatic detection technology.

\section{Ethics approval and consent to participate}

Not applicable

\section{Consent for publication}

Not applicable

\section{Competing interests}

The authors declare that they have no competing interests. And all authors have seen the manuscript and approved to submit to your journal. We confirm that the content of the manuscript has not been published or submitted for publication elsewhere.

\section{Publisher's Note}

Springer Nature remains neutral with regard to jurisdictional claims in published maps and institutional affiliations.

\section{Author details}

${ }^{1}$ College of Mechanical and Electrical Engineering, Nanning University, No.8 Lonting Road, Nannin 530200, China. ${ }^{2}$ Guangxi Quality Technical Engineering School, No.8 Lonting Road, Nannin 530200, China.

Received: 16 April 2018 Accepted: 25 June 2018

Published online: 21 August 2018

References

1. A Guo, H Zhou, Teaching reform for automatic control principle. J Electr Electron Educ 36(1), 11-12 (2014)

2. Y Liu, P Wang, L Liao, et al., LOR controller optimization for double inverted pendulum based on improved artificial bee colony algorithm. Comput Measur Control 22(9), 2820-2822 (2014)

3. X Wang, W Chen, X Zhao, Overview of swing-up and stability control of the inverted pendulum system. Tech Autom Appl 34(11), 5-9 (2015)

4. Q Wang, H Huang, Symbol-type adaptive fuzzy control on inverted pendulum system. Commun Eng Des 35(3), 1016-1020 (2014)

5. Z Zhang, X Wei, S Wang, Study and design of the rotational inverted pendulum based on 52 microcontroller. J Langfang Teach Coll 14(4), 49-52 (2014)

6. Z Wu, Real-time simulation of control system based on RTX, Simulink and LabWindows/CVI. Electron Des. Eng 22(13), 44-47 (2014)

7. Q Song, D Li, The design and stability study of double inverted pendulum controller. Comput Simul 32(4), 305-309 (2015)

8. S Zhu, X Wang, W Liu, Robust adaptive repetitive control for periodically time-varying systems. Acta Automat Sin 40(11), 2391-2403 (2014)

9. D Qiao, Y Zhang, L Shi, Optimization design of binocular stereo vision sensor based on genetic algorithm. Journal of Natural Science of Hunan Normal University 37(6), 53-56 (2014)

10. Y Wang, D Wang, LQR control method for double-inverted pendulum system based on Kalman filter. J Qingdao Univ 30(3), 21-26 (2015)

\section{Submit your manuscript to a SpringerOpen ${ }^{\circ}$ journal and benefit from:}

- Convenient online submission

Rigorous peer review

- Open access: articles freely available online

- High visibility within the field

- Retaining the copyright to your article

Submit your next manuscript at $\gg$ springeropen.com 